\title{
Risk Factors Associated with Heart Valve Thrombosis in Patients with Prosthetic Heart Valve Dysfunction
}

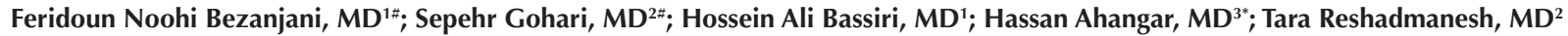 \\ ${ }^{1}$ Department of Cardiology, Rajaie Heart Center, Iran University of Medical Sciences, Tehran, Iran \\ ${ }^{2}$ School of Medicine, Zanjan University of Medical Sciences, Zanjan, Iran \\ ${ }^{3}$ Department of Cardiology, Mousavi Hospital, School of Medicine, Zanjan University of Medical Sciences, Zanjan, Iran
}

\begin{abstract}
Background: Thrombotic and thromboembolic events are important causes of mortality and morbidity in patients with prosthetic heart valve. The aim of this study is to evaluate the factors that may contribute to prosthetic heart valve thrombosis.

Methods: This was a cross-sectional study in Rajaie Heart Center on patients with prosthetic heart valve malfunction, within a year. According to the echocardiographic and fluoroscopic findings, the patients were divided into two groups (thrombosis and non-thrombosis groups). The patients' demographic, clinical and laboratory data were recorded and analyzed with SPSS software. Results: A total of 142 patients participated in this study. Ninety-four patients $(66.2 \%)$ were diagnosed with thrombosis. There was a significant relationship between thrombosis and inadequate anti-coagulation (international normalized rati [INR] $<2.5)$ (odds ratio [OR]: $4.15,95 \% \mathrm{Cl}: 1.98-9.87, P=0.003)$, history of infection (OR: $12.81,95 \% \mathrm{Cl}: 3.52-19.02, P<0.001)$, prothrombin time (PT) check interval (OR: 2.38, 95\% Cl: 1.63-8.47, $P=0.019$ ), atrial fibrillation (AF) rhythm (OR: 3.96, 95\% Cl: 1.75-8.09, $P$ $=0.019$ ), and plasma fibrinogen level (OR: $6.90,95 \% \mathrm{Cl}: 2.58-14.69)$.

Conclusion: Based on this study, inadequate anti-coagulation, AF rhythm, recent infection and plasma fibrinogen level were the factors most contributing to prosthetic valve thrombosis. As there were many cases of thrombosis in patients with history of infection, this factor can be considered for risk assessment in prosthetic valve.

Keywords: Heart valve prosthesis, Risk factors, Thrombosis

Cite this article as: Noohi Bezanjani F, Gohari S, Bassiri HA, Ahangar H, Reshadmanesh T. Risk factors associated with heart valve thrombosis in patients with prosthetic heart valve dysfunction. Arch Iran Med. 2020;23(9):600-604. doi: 10.34172/aim.2020.70.
\end{abstract}

Received: July 3, 2019, Accepted: March 9, 2020, ePublished: September 1, 2020

\section{Introduction}

An average of 350000 heart valves are replaced worldwide every year. ${ }^{1}$ The main objective of valve replacement is to expand life expectancy and improve life quality in patients who have significant symptoms. ${ }^{2}$ One of the important steps in treatment of these patients is sufficient administration of anticoagulants to prevent thrombus formation on the valve. ${ }^{3}$ The incidence of prosthetic heart valve thrombosis depends on the type of the prosthetic valve. In mechanical valves, the risk of thrombosis is higher compared to biological valves. There is an incidence rate of thrombosis of approximately $0.1 \%$ to $5.7 \%$ in mechanical heart valves and $0.03 \%$ in biological heart valves, reported every year. Also, it is reported that thrombosis occurs by a rate between 0.1 to $6.0 \%$ for each year of patient's age, in the mitral and the aortic valves and up to $20 \%$ in the tricuspid valve. ${ }^{4}$ Diagnostic techniques including echocardiography, fluoroscopy, and therapeutic measures involving re-surgery, thrombolytic therapy, or anti-coagulant treatments impose significant costs on individuals and the whole community. ${ }^{5,6}$ Prosthetic Valve Thrombosis (PVT) results in valve malfunction, thromboembolic events, the clot serving as a nest for viral or bacterial growth, etc. ${ }^{7}$ This study aims to assess the risk factors and incidence of prosthetic valve thrombosis in patients with prosthetic valve malfunction.

\section{Materials and Methods}

Study Design and Participants

This was a cross-sectional study in Rajaie Heart Centre on patients with prosthetic heart valve malfunction. Depending on the purpose and type (census) of the study, all patients (referred from other hospitals, emergency admittance and routine visits) with valve malfunction (symptomatic or asymptomatic changes in the prosthetic valve's hemodynamic status on echocardiographic assessment, including stenosis and regurgitation) from April 2015 to April 2016 were included in the study. The criteria for excluding patients were: 1) missing data obtained by laboratory tests or interview, and 2) the patient not consenting to the study.

In case of clinical suspicion for prosthetic valve thrombosis, transthoracic echocardiography was performed in advance. Based on the leaflet mobility and gradient, hemodynamic status, and identification of thrombus, the trans-esophageal echocardiography was performed in 
the absence of contraindications. The considered signs of thrombosis on echocardiography were restriction of leaflet mobility or observing echogenic thrombotic mass on the leaflet or valve ring. If thrombosis was diagnosed, fluoroscopy was performed to confirm the restriction of the related leaflet movement. ${ }^{8}$

\section{Variables}

The patients' demographic data, in addition to medical and drug history, were obtained through interviews. The interviewer was blinded to the other data of the patients. Then, a blood sample was collected aseptically by antecubital puncture, followed by a urine test. Laboratory measurements included the international normalized ratio (INR), C-reactive protein (CRP), erythrocyte sedimentation rate (ESR), plasma fibrinogen level and proteinuria. Researchers who collected either the interview or laboratory data were blinded to the other data.

\section{Statistical Analysis}

The collected data were analyzed using the IBM SPSS Statistics software (version 16.0, SPSS, Chicago, Illinois). Chi-square test was used to compare qualitative variables. Quantitative variables were compared using Student's $t$ test. Significance differences were shown with odds ratio $(95 \% \mathrm{CI})$ computed using chi-square test. Logistic regression was used to assess the relationship between the variables and PVT and determining the confounder effects. $P$ values $<0.05$ were considered significant.

\section{Results}

Demographic Data and Medical History

A total of 154 eligible patients participated in the study. Twelve patients were excluded due to lack of co-operation or missing data. Finally, data from 142 patients were analyzed. Ninety-four patients $(66.2 \%)$ were diagnosed with thrombosis and $48(33.8 \%)$ were without thrombosis (infective endocarditis, para-valvular leakage, valve deterioration). One hundred and two patients (71.8\%) had dyspnea at the time of presentation which was the most prevalent symptom. Thirteen patients (9.2\%) had palpitation, 13 (9.2\%) had CVA (cerebrovascular accidents) and TIA (transient ischemic attack) and 1 $(0.7 \%)$ experienced syncope. None of the patients were addicted to drugs or alcohol but $19(13.4 \%)$ used to smoke cigarettes. Ninety-four patients $(66.2 \%)$ were females and $48(33.8 \%)$ were males with an average (mean \pm SD) age of $49.8 \pm 13.13$ years. Seventy-five patients $(52.8 \%)$ had a history of an infectious disease other than endocarditis in the month preceding admission [69 (92\%) in the thrombosis versus $6(8 \%)$ in the non-thrombosis group]: among them, 54 (72\%) had upper respiratory infection (OR: 19.320, 95\% CI: 7.323-50.969). According to the history of prothrombin time (PT) check intervals, 57 patients $(60.7 \%)$ with thrombosis versus $3(6.2 \%)$ without thrombosis had erratic PT check. In other words, the nonthrombosis group had considerably regular PT check intervals $(P<0.001)$ (Table 1$)$. Logistic regression showed that history of recent infection and PT check interval were

Table 1. Patients' Demographic Characteristics and Medical History

\begin{tabular}{|c|c|c|c|c|c|c|}
\hline \multirow{2}{*}{ Variables } & \multirow{2}{*}{ Thrombosis $(n=94)$, No. $(\%)$} & \multirow{2}{*}{ Non-Thrombosis $(n=48)$, No. $(\%)$} & \multirow{2}{*}{$P$ Value } & \multirow{2}{*}{ OR } & \multicolumn{2}{|c|}{$\mathrm{Cl}(95 \%)$} \\
\hline & & & & & Lower & Upper \\
\hline \multicolumn{7}{|l|}{ Gender } \\
\hline Female & $65(69.1)$ & $29(60.4)$ & \multirow{2}{*}{0.298} & \multirow{2}{*}{1.468} & \multirow{2}{*}{0.711} & \multirow{2}{*}{3.033} \\
\hline Male & $29(30.9)$ & 19 (39.6) & & & & \\
\hline \multicolumn{7}{|c|}{ PT check interval } \\
\hline$<1$-month & $2(2.1)$ & $6(12.5)$ & \multirow[t]{3}{*}{$<0.001$} & 1.09 & 0.95 & 1.25 \\
\hline Monthly & $35(37.2)$ & $39(81.3)$ & & Base & - & - \\
\hline Erratic & $57(60.7)$ & $3(6.2)$ & & 8.67 & 2.88 & 26.11 \\
\hline \multicolumn{7}{|c|}{ Recent infection } \\
\hline Yes & $69(73.4)$ & $6(12.5)$ & \multirow[t]{2}{*}{$<0.001$} & \multirow[t]{2}{*}{19.320} & \multirow[t]{2}{*}{7.323} & \multirow[t]{2}{*}{50.969} \\
\hline No & $25(26.6)$ & $42(87.5)$ & & & & \\
\hline \multicolumn{7}{|c|}{ Aspirin $(80)+$ warfarin } \\
\hline Yes & $36(38.3)$ & $34(70.8)$ & \multirow[t]{2}{*}{$<0.001$} & \multirow[t]{2}{*}{0.256} & \multirow[t]{2}{*}{0.121} & \multirow[t]{2}{*}{0.540} \\
\hline No & $58(61.7)$ & $14(29.2)$ & & & & \\
\hline \multicolumn{7}{|l|}{ Season } \\
\hline Spring & $19(20.2)$ & $14(29.2)$ & \multirow{4}{*}{0.811} & \multirow{4}{*}{-} & \multirow{4}{*}{-} & \multirow{4}{*}{-} \\
\hline Summer & $17(18.1)$ & $13(27.1)$ & & & & \\
\hline Fall & $23(24.5)$ & $8(16.6)$ & & & & \\
\hline Winter & $35(37.2)$ & $13(27.1)$ & & & & \\
\hline \multicolumn{7}{|c|}{ Replaced valve number } \\
\hline 1 & $57(60.6)$ & $39(81.3)$ & \multirow{3}{*}{0.004} & \multirow{3}{*}{ - } & \multirow{3}{*}{ - } & \multirow{3}{*}{-} \\
\hline 2 & $36(38.3)$ & $9(18.7)$ & & & & \\
\hline 3 & $1(1.1)$ & $0(0.0)$ & & & & \\
\hline
\end{tabular}

PT, prothrombin time; $\mathrm{OR}$, odds ratio; $\mathrm{Cl}$, confidence interval. 
the risk factors most contributing to thrombosis (OR: 12.81, 95\% CI: 3.52-19.02, $P<0.001$ and OR: $2.38,95 \%$ CI: $1.63-8.47, P=0.019$, respectively) (Table 2 ).

\section{Clinical Findings}

One hundred and twenty patients (84.5\%) had normal metallic sound and $22(15.5 \%)$ had reduced metallic sound. All of the reduced sounds pertained to the thrombosis group and the difference was significant (OR: 1.66, 95\% CI: 1.44-1.92, $P<0.001)$. Among patients with atrial fibrillation (AF), $52(77.61 \%)$ were in the thrombosis group and $15(22.39 \%)$ were in the nonthrombosis group, indicating a significant difference $(P=$ 0.008) (Table 3).

Also, a significant relationship was observed between AF rhythm and thrombosis (OR: 3.96, 95\% CI: 1.75-8.09, $P$ $=0.018)$. Additional information regarding the effects of these factors is illustrated in Table 2.

\section{Laboratory Measurements}

According to Table 4, the mean INR level was 1.8 in the first group and 2.3 in the second group. Thrombotic events were observed with significantly higher frequency in the patients with INR $\leq 2.5(P<0.001)$. Analysis showed a direct relationship between the INR level of less than 2.5 and thrombotic events (OR: 4.15, 95\% CI: 1.98 9.87, $P=0.003)$. The CRP level increased in 66 patients (46.5\%), 55 of whom (83.33\%) were in the thrombosis group. There was a significant difference between the two groups based on the CRP level (OR: 2.55, 95\% CI: 1.47-4.40, $P<0.001)$. In the urine analysis, 37 patients (39.4\%) had $1+$ proteinuria and 7 patients $(7.4 \%)$ had $2+$ proteinuria in the thrombosis group. In the nonthrombosis group, no patient had $2+$ proteinuria. There was a significant difference between the two groups based on the proteinuria level $(P<0.001)$ (Table 4$)$. According to Table 2, the effects of CRP and proteinuria were not significant in logistic regression. Although there was a significant difference between the two groups, they were considered as confounder factors (Table 2).

\section{Discussion}

Valvular heart disease is prevalent in developing countries. Surgical replacement is an advanced therapy for this disease, with optimal long-term results. One of the most important complications of valve replacement surgery

Table 2. Relationship Between Risk Factors and Thrombosis in Logistic Regression Analysis

\begin{tabular}{|c|c|c|c|c|}
\hline \multirow{2}{*}{ Risk Factors } & \multirow{2}{*}{$P$ value } & \multirow{2}{*}{ OR } & \multicolumn{2}{|c|}{$\mathrm{Cl}(95 \%)$} \\
\hline & & & Lower & Upper \\
\hline PT Check Interval & 0.019 & 2.38 & 1.63 & 8.47 \\
\hline Recent infection & $<0.001$ & 12.81 & 3.52 & 19.02 \\
\hline Concurrent use of aspirin and warfarin & 0.138 & 0.211 & 0.027 & 1.65 \\
\hline Replaced valve number & 0.282 & 2.47 & 0.292 & 5.51 \\
\hline CRP & 0.100 & 2.11 & 0.656 & 4.56 \\
\hline Proteinuria & 0.180 & 0.16 & 0.011 & 2.32 \\
\hline Plasma fibrinogen & 0.006 & 6.90 & 2.58 & 14.69 \\
\hline Fluoroscopy & 0.264 & 2.05 & 0.295 & 3.54 \\
\hline AF rhythm & 0.018 & 3.96 & 1.75 & 8.09 \\
\hline INR level & 0.003 & 4.15 & 1.98 & 9.87 \\
\hline
\end{tabular}

PT, prothrombin time; CRP, C-reactive protein; AF, atrial fibrillation; INR, international normalized ratio; OR, odds ratio; $\mathrm{Cl}$, confidence interval.

Table 3. Patients' Clinical and Imaging Findings

\begin{tabular}{|c|c|c|c|c|c|c|}
\hline \multirow{2}{*}{ Variables } & \multirow{2}{*}{ Thrombosis $(n=94)$, No. $(\%)$} & \multirow{2}{*}{ Non-Thrombosis $(n=48)$, No. $(\%)$} & \multirow{2}{*}{$P$ Value } & \multirow{2}{*}{ OR } & \multicolumn{2}{|c|}{$\mathrm{Cl}(95 \%)$} \\
\hline & & & & & Lower & Upper \\
\hline \multicolumn{7}{|c|}{ Metallic sound } \\
\hline Decreased & $22(23.4)$ & $0(0.0)$ & $<0.001$ & 1.66 & 1.44 & 1.92 \\
\hline Normal & $72(76.6)$ & $48(100)$ & & & & \\
\hline \multicolumn{7}{|l|}{ Rhythm } \\
\hline Sinus & $42(44.7)$ & $33(68.7)$ & 0.008 & 1.77 & 1.12 & 2.79 \\
\hline $\mathrm{AF}$ & $52(55.3)$ & $15(31.3)$ & & & & \\
\hline \multicolumn{7}{|l|}{ Fluoroscopy } \\
\hline Limited & $49(52.1)$ & $6(12.5)$ & $<0.001$ & 4.17 & 1.92 & 9.03 \\
\hline Normal & $45(47.9)$ & $42(87.5)$ & & & & \\
\hline \multicolumn{7}{|l|}{ LVEF } \\
\hline$<35 \%$ & $15(15.9)$ & $5(10.4)$ & & 1.44 & 0.68 & 3.02 \\
\hline $36 \%-50 \%$ & $69(73.4)$ & $36(75)$ & 0.44 & 1.04 & 0.89 & 1.22 \\
\hline$>50 \%$ & $10(10.6)$ & $7(14.6)$ & & Base & 一 & 一 \\
\hline
\end{tabular}

$\mathrm{AF}$, atrial fibrillation; LVEF, left ventricular ejection fraction; $\mathrm{OR}$, odds ratio; $\mathrm{Cl}$, confidence interval. 
Table 4. Laboratory Measurements

\begin{tabular}{|c|c|c|c|c|c|c|}
\hline \multirow{2}{*}{ Variables } & \multirow{2}{*}{ Thrombosis $(n=94)$, No. $(\%)$} & \multirow{2}{*}{ Non-Thrombosis $(n=48)$, No. $(\%)$} & \multirow{2}{*}{$P$ Value } & \multirow{2}{*}{ OR } & \multicolumn{2}{|c|}{$\mathrm{Cl}(95 \%)$} \\
\hline & & & & & Lower & Upper \\
\hline \multicolumn{7}{|l|}{ INR } \\
\hline$<1.5$ & $34(36.2 \%)$ & $5(10.4 \%)$ & \multirow{4}{*}{$<0.001$} & 3.32 & 1.51 & 7.31 \\
\hline $1.5-2.5$ & $49(52.1 \%)$ & $26(54.2 \%)$ & & 2.91 & 1.19 & 7.12 \\
\hline$>2.5$ & $11(11.7 \%)$ & $17(35.4 \%)$ & & Base & - & - \\
\hline Mean & 1.8 & 2.35 & & - & - & - \\
\hline \multicolumn{7}{|l|}{ CRP } \\
\hline Normal & $39(41.5 \%)$ & $37(77.1 \%)$ & \multirow{2}{*}{$<0.001$} & \multirow{2}{*}{2.55} & \multirow{2}{*}{1.47} & \multirow{2}{*}{4.40} \\
\hline Increased & $55(58.5 \%)$ & $11(22.9 \%)$ & & & & \\
\hline \multicolumn{7}{|l|}{ ESR } \\
\hline Normal & $80(85.1 \%)$ & $42(87.5 \%)$ & \multirow{2}{*}{0.698} & \multirow{2}{*}{1.19} & \multirow{2}{*}{0.49} & \multirow{2}{*}{2.90} \\
\hline Increased & $14(14.9 \%)$ & $6(12.5 \%)$ & & & & \\
\hline \multicolumn{7}{|l|}{ Proteinuria } \\
\hline Normal & $50(53.2 \%)$ & $41(85.4 \%)$ & \multirow{3}{*}{$<0.001$} & Base & - & - \\
\hline+1 & $37(39.4 \%)$ & $7(14.6 \%)$ & & 2.91 & 1.41 & 6.03 \\
\hline+2 & $7(7.4 \%)$ & $0(0.0 \%)$ & & 1.54 & 1.15 & 3.14 \\
\hline \multicolumn{7}{|c|}{ Plasma fibrinogen } \\
\hline Normal & $46(48.9 \%)$ & $47(97.9 \%)$ & \multirow{2}{*}{$<0.001$} & \multirow{2}{*}{24.51} & \multirow{2}{*}{3.48} & \multirow[t]{2}{*}{172.19} \\
\hline Increased & $48(51.1 \%)$ & $1(2.1 \%)$ & & & & \\
\hline
\end{tabular}

INR, international normalized ratio; CRP, C-reactive protein; ESR, erythrocyte sedimentation rate; OR, odds ratio; $\mathrm{Cl}$, confidence interval.

is dysfunction due to valve thrombosis which can lead to thromboembolic events and fatal stroke. ${ }^{4}$ According to Table 4, inadequate anti-coagulation at the time of admission had a strong relationship with thrombosis. Similarly, as mentioned in other studies, the cause of thrombosis in prosthetic valves is often due to insufficient anticoagulation, which is directly related to the patients' culture and their awareness regarding correct use of anticoagulant drugs. ${ }^{4,9}$ The most common symptom at the time of admission was dyspnea, and later with other findings such as palpitation, CVA, TIA, decreased prosthetic valve sounds and syncope. These findings are consistent with another study conducted by Bonou et al. ${ }^{10}$ In this study, the highest rate of thrombosis observed was in winter in the months of January and February (Table 1). Pham et $\mathrm{al}^{11}$ reported that PVT has a higher prevalence during winter compared to other seasons. It seems that this caused by the change in plasma fibrinogen levels in different seasons. In winter, plasma fibrinogen levels increase. Also, the increase in plasma viscosity in this season may be the cause for an increase in thrombosis. Our study also found a significant relationship between plasma fibrinogen levels and thrombosis which increased the incidence of thrombosis in those with higher plasma fibrinogen levels. This also confirms all above. ${ }^{12}$ In this study, AF rhythm on ECG had a direct and significant relationship with valve thrombosis. Dürrleman et al. ${ }^{13}$ reported that AF rhythm was observed in $45 \%$ of patients with PVT. Furthermore, Cáceres-Lóriga et $\mathrm{al}^{14}$ reported that this rhythm was present in 56\% of patients with PVT and there was a significant relation between $\mathrm{AF}$ and thrombosis, which is consistent with our study. The results show a significant relationship between history of infection, particularly upper respiratory infection in the month preceding admission, and PVT. Since none of the patients with previous infections, especially upper respiratory tract infection, were diagnosed with endocarditis, it can be argued that the presence of upper respiratory tract infections without direct involvement of cardiac tissue, could be an independent factor in development of thrombotic events in prosthetic valves. We did not find any study addressing this issue in the databases. In this study, the CRP level was considered as a confounder factor for PVT, but Gürsoy et $\mathrm{al}^{15}$ reported that the increased CRP level contributed to the prosthetic mitral valve thrombosis. A possible reason why this factor was considered a confounding factor in this study is that $\mathrm{CRP}$ is an acute phase reactant and can change quickly in conditions other than valve dysfunction. We also found proteinuria level as a confounding factor.

One of the limitations of our study was the presence of infection before the patients' admission as a subjective item. We were not able to confirm the presence of infection with para-clinical tests, and we could not differentiate the bacterial or the viral nature of the infection. Another limitation was that our study was conducted in a single clinical heart center. Consequently, the results may not have enough power or validity to be generalized. Also, in this study, we did not evaluate the effect of the time lapse between valve surgery and the dysfunction which could be an important factor; this needs to be considered in future studies.

In conclusion, based on the results of our study, it may be 
concluded that inadequate anti-coagulation, AF rhythm, recent infection and plasma fibrinogen level are the factors most contributing to prosthetic valve thrombosis. Due to the high rates of thrombosis in patients with history of recent infection, this factor can be considered as a factor in prosthetic valve risk assessment, requiring further investigations in larger populations.

\section{Authors' Contribution}

FNB: made total concept, designing the study and manuscript review. HAB: definition of intellectual content and design the study. SG: searching the literatures, manuscript preparation, definition of intellectual content and data curation. HA: designing the study, performing clinical section, data acquisition and manuscript editing. TR: data statictical analysis.

\section{Conflict of Interest Disclosures}

Hereby the authors declare no conflict of interest.

\section{Ethical Statement}

The study was conducted in accordance with the principles of the declaration of Helsinki and approved by the ethic committee of Iran University of Medical Sciences. All patients provided their written informed consent.

\section{Founding}

None.

\section{Acknowledgements}

We would like to express our special thanks to all of patients for their kind cooperation in this study. Moreover we thank Mr. Naser Reshadmanesh for his noteworthy consultations regarding the statistical analysis. The abstract of this study has been presented (oral presentation) at the 23rd European Heart Disease and Failure Congress, on February 19-21, 2018, in Paris, France.

\section{References}

1. Zilla P, Brink J, Human P, Bezuidenhout D. Prosthetic heart valves: catering for the few. Biomaterials. 2008;29(4):385406. doi: 10.1016/j.biomaterials.2007.09.033.

2. Nishimura RA, Otto CM, Bonow RO, Carabello BA, Erwin JP, Fleisher LA, et al. AHA/ACC focused update of the 2014 AHA/ ACC guideline for the management of patients with valvular heart disease: a report of the American College of Cardiology/ American Heart Association Task Force on Clinical Practice Guidelines. J Am Coll Cardiol. 2017;70(2):252-89. doi: 10.1016/j.jacc.2017.03.011.
3. lung B, Rodés-Cabau J. The optimal management of antithrombotic therapy after valve replacement: certainties and uncertainties. Eur Heart J. 2014;35(42):2942-9. doi: 10.1093/ eurheartj/ehu365.

4. Dangas GD, Weitz JI, Giustino G, Makkar R, Mehran R. Prosthetic heart valve thrombosis. J Am Coll Cardiol. 2016;68(24):2670-89. doi: 10.1016/j.jacc.2016.09.958.

5. Krishnan S. Prosthetic heart valve thrombosis: diagnosis and newer thrombolytic regimes. J Pract Cardiovasc Sci. 2016;2(1):7-12. doi: 10.4103/2395-5414.182993.

6. Biteker M, Altun I, Basaran O, Dogan V, Yildirim B, Ergun G. Treatment of prosthetic valve thrombosis: current evidence and future directions. J Clin Med Res. 2015;7(12):932-6. doi: 10.14740/jocmr2392w.

7. Roudaut R, Serri K, Lafitte S. Thrombosis of prosthetic heart valves: diagnosis and therapeutic considerations. Heart. 2007;93(1):137-42. doi: 10.1136/hrt.2005.071183.

8. Otto CM. The practice of clinical echocardiography. 5th ed. USA: Elsevier Health Sciences; 2017: 455-480.

9. Salamon J, Munoz-Mendoza J, Leibelt JJ, Taub CC. Mechanical valve obstruction: Review of diagnostic and treatment strategies. World J Cardiol. 2015;7(12):875-81. doi: 10.4330/ wjc.v7.i12.875.

10. Bonou M, Lampropoulos K, Barbetseas J. Prosthetic heart valve obstruction: thrombolysis or surgical treatment? Eur Heart J Acute Cardiovasc Care. 2012;1(2):122-7. doi: 10.1177/2048872612451169.

11. Pham N, Zaitoun H, Mohammed TL, DeLaPena-Almaguer E, Martinez F, Novaro GM, et al. Complications of aortic valve surgery: manifestations at CT and MR imaging. Radiographics. 2012;32(7):1873-92. doi: 10.1148/rg.327115735.

12. Piper C, Hering D, Horstkotte D. Prosthetic valve thrombosis: predisposition and diagnosis. Eur Heart J Suppl. 2001;3(suppl_Q):Q16-Q21. doi: 10.1016/S1520765X(01)90037-9.

13. Dürrleman N, Pellerin $M$, Bouchard $D$, Hébert $Y$, Cartier R, Perrault LP, et al. Prosthetic valve thrombosis: twenty-year experience at the Montreal Heart Institute. J Thorac Cardiovasc Surg. 2004;127(5):1388-92. doi: 10.1016/j.jtcvs.2003.12.013.

14. Cáceres-Lóriga FM, Pérez-López H, Santos-Gracia J, MorlansHernandez K. Prosthetic heart valve thrombosis: pathogenesis, diagnosis and management. Int J Cardiol. 2006;110(1):1-6. doi: 10.1016/j.ijcard.2005.06.051.

15. Gürsoy OM, Karakoyun $S$, Kalçık $M$, Gökdeniz $T$, Yesin $M$, Gündüz $S$, et al. Usefulness of novel hematologic inflammatory parameters to predict prosthetic mitral valve thrombosis. Am J Cardiol. 2014;113(5):860-4. doi: 10.1016/j. amjcard.2013.11.029. 